\title{
Self-assembled Nano-polycatenanes
}

Sougata Datta ${ }^{1}$, Yasuki Kato ${ }^{2}$, Seiya Higashiharaguchi ${ }^{2}$, Keisuke Aratsu $^{2}$, Atsushi Isobe ${ }^{2}$, Takuho Saito $^{2}$, Deepak D. Prabhu ${ }^{1}$, Yuichi Kitamoto ${ }^{3}$, Martin J. Hollamby ${ }^{4}$, Andrew J. Smith ${ }^{5}$, Robert

Dagleish $^{6}$, Najet Mahmoudi ${ }^{6}$, Luca Pesce ${ }^{7}$, Claudio Perego ${ }^{7}$, Giovanni M. Pavan ${ }^{7,8} \&$ Shiki Yagai $^{1,2,3 *}$

${ }^{1}$ Department of Applied Chemistry and Biotechnology, Graduate School of Engineering, Chiba University, 1-33 Yayoi-cho, Inage-ku, Chiba 263-8522, Japan.

${ }^{2}$ Division of Advanced Science and Engineering, Graduate School of Science and Engineering, Chiba University, 1-33 Yayoi-cho, Inage-ku, Chiba 263-8522, Japan.

${ }^{3}$ Institute for Global Prominent Research (IGPR), Chiba University, 1-33 Yayoi-cho, Inage-ku, Chiba 263-8522, Japan.

${ }^{4}$ School of Chemical and Physical Sciences, Keele University, Keele, Staffordshire ST55BG, UK.

${ }^{5}$ Diamond Light Source Ltd., Diamond House, Harwell Science and Innovation Campus, Didcot, Oxon OX110DE, UK.

${ }^{6}$ ISIS Pulsed Neutron and Muon Source, Rutherford Appleton Laboratory, Chilton, Oxon, OX11 0QX, UK.

${ }^{7}$ Department of Innovative Technologies, University of Applied Sciences and Arts of Southern Switzerland, Galleria 2, Via Cantonale 2c, CH-6928 Manno, Switzerland.

${ }^{8}$ Department Department of Applied Science and Techology, Politecnico di Torino, Corso Duca degli Abruzzi 24, 10129 Torino, Italy.

E-mail: yagai@faculty.chiba-u.jp; Fax: +81-(0)43-290-3401; Tel: +81-(0)43-290-3169 
Mechanical interlocking of molecules (catenation) is a nontrivial challenge in modern synthetic chemistry and materials science, especially in cases where biologically inspired features are targeted $^{1,2}$. One strategy to achieve catenation is the design of pre-annular molecules that are capable of both efficient cyclization and of pre-organizing another precursor to engage in subsequent interlocking ${ }^{3-9}$. This task is particularly difficult when the annular target is composed of a large ensemble of molecules, i.e., when the target is a supramolecular assembly. However, the construction of such unprecedented assemblies would enable the visualization of nontrivial nanotopologies through microscopy techniques, which would not only satisfy academic curiosity, but also pave the way to materials with nanotopology-derived properties. Herein, we report the synthesis of such a type of nanotopology using fibrous supramolecular assemblies with intrinsic curvature. Using a solvent-mixing strategy, we kinetically organized a molecule that can elongate into toroids with a radius of ca. $13 \mathrm{~nm}$. Atomic force microscopy (AFM) on the resulting nanoscale toroids revealed a high percentage of oligo-catenation, which is sufficient to yield “nanolympiadane"10. Spectroscopic and theoretical studies suggested that this unusually high degree of catenation stems from the secondary nucleation of the precursor molecules around the toroids. By modifying the self-assembly protocol to promote ring-closure and secondary nucleation, a maximum catenation number of 22 was confirmed by AFM.

A crucial requirement for the bottom-up creation of an interlocked nanotopology is a molecular assembly system that can reproducibly provide annular nanostructures with a uniform diameter and sufficient inner void space. The topological supramolecular polymers that our group has explored over the past few years ideally satisfy this principal requirement ${ }^{11}$. We have developed a variety of barbiturated $\pi$-conjugated molecules that can form six-membered supermacrocycles, which we have termed "rosettes", through hydrogen bonds (Fig. 1a). The formation of these rosettes is coupled to the nucleation of supramolecular polymerization via $\pi-\pi$ stacking of the rosettes with translational and 
rotational displacement (Fig. 1b) ${ }^{12,13}$. The repetition of this specific rosette stacking induces a uniform, intrinsic curvature (radius: 7-13 nm; Fig. 1a), which eventually leads to the formation of supramolecular polymers with unprecedented topologies, including toroids ${ }^{12-14}$, helicoids ${ }^{15,16}$, and random coils (Fig. $1 c)^{16}$. The topology of the polymers depends on the kinetics of the supramolecular polymerization, which can be controlled via the preparation protocol.

Based on the following observations, we chose 1 (Fig. 1a) as the monomer to realize unprecedented nanotopologies. Under thermodynamic control, e.g., slow cooling $\left(\sim 1.0 \mathrm{~K} \mathrm{~min}^{-1}\right)$ of a hot methylcyclohexane $(\mathrm{MCH})$ solution of $\mathbf{1}$, molecules of $\mathbf{1}$ organizes into helicoidal fibers through temperature-regulated supramolecular polymerization ${ }^{16}$. As increasing the cooling rate to $10 \mathrm{~K} \mathrm{~min}^{-1}$, the topologies of the resulting polymers gradually change to randomly coiled structures. Upon abrupt cooling, i.e., thermal "quenching"17, we obtained a small amount of toroids as kinetically trapped species that were formed via a ring-closing pathway (Supplementary Fig. 1). Ring-closure of the supramolecular polymers is further facilitated by promoting kinetic control of the polymerization via mixing different types of solvents. In this protocol, monomers concentrated in a good (polar) solvent $(100 \mu \mathrm{L})$ were injected vigorously into $900 \mu \mathrm{L}$ of a poor (less polar) solvent (Fig. 2a). As the monomer solution diffuses, the environment surrounding the monomers becomes less polar, initiating supramolecular polymerization. When chloroform and $\mathrm{MCH}$ were used as good and poor solvent, respectively, an atomic force microscopy (AFM) analysis (Supplementary Method and Supplementary Fig. 2) revealed that $44 \%$ of the monomers (total monomer concentration, $c_{\mathrm{T}}=1 \times 10^{-4} \mathrm{M}$ ) were kinetically trapped in toroid form (Fig. 2b,c and Supplementary Fig. 3a). It is noteworthy that 3\% of the total toroids were obtained as geometrically interlocked nano-[2]catenanes under these conditions (Fig. 2b-d and Supplementary Fig. 3b). 
The result that $3 \%$ of the toroids were obtained as nano-[2]catenanes motivated us to study another requirement for interlocked nanotopologies: templated supramolecular polymerization. Since simply increasing the monomer concentration did not improve the nanocatenane yield (Supplementary Fig. 4), the formation of the nanocatenanes cannot be explained by the stochastic interlocking of growing toroids. Historically, the poor yield $(<1 \%)$ obtained by stochastic catenation ${ }^{8}$ during cyclization reactions was improved by covalent template strategies, but at the cost of a much longer synthetic route. ${ }^{18}$ Sauvage and coworkers first overcame all the previous limitations and revolutionized the synthesis of catenanes by discovering template directed synthetic strategy, wherein annular and proannular components were preorganized using metal-ligand interactions ${ }^{7}$. The scope of this so-called template-directed approach has been expanded by other researchers using $\pi$-donor-acceptor interactions ${ }^{6}$, hydrogen bonding ${ }^{5}$, and the hydrophobic effect ${ }^{3,4}$. In our supramolecular polymers, which are covered with linear alkyl chains, preorganization of monomers via van der Waals interactions could potentially occur at the surface of the preformed toroids to facilitate heterogeneous nucleation (secondary nucleation; Fig. 1d) ${ }^{19}$. If this type of templated nucleation were to occur at the interior surface of the toroids, subsequent elongation and ring-closure of the resulting polymers would result in nano-[2]catenanes. On the other hand, if the secondarily nucleated fibers fail to close into toroids, the elongation of the open-ended fibers would lead to pseudorotaxanes (Fig. 2e and Supplementary Discussion 1).

Using a seeding experiment with separately prepared toroids ${ }^{20-22}$, we assessed whether the secondary nucleation could occur in a solution of $\mathbf{1}$. For this purpose, we initially established a method to isolate toroids from a complex mixture of supramolecular polymers with various topologies by exploiting their kinetic stability due to their lack of termini and relatively small size ${ }^{23}$. In brief, the open-ended short coils in a mixture of supramolecular polymers were selectively reorganized into elongated helical fibers with lengths $>1 \mu \mathrm{m}$ by thermal annealing (Fig. $2 \mathrm{f}$ and Supplementary Fig. 5a). The resulting elongated 
fibers could be readily filtered out from the toroids using a membrane filter with a pore size of $200 \mathrm{~nm}$ (Fig. $2 \mathrm{f}$ and Supplementary Fig. 5b). The toroid structure in solution was characterized by small-angle X-ray and neutron scattering (SAXS, SANS) measurements, whose results showed good agreement with the results of AFM measurements and those of a model based on rosettes (Fig. 2i,j, Supplementary Figs. 6-8 and Supplementary Table 1$)^{12,24}$. The toroid dimensions obtained by fitting analysis (toroid radius, $R=12.5 \mathrm{~nm}$; cross-sectional radius excluding alkyl shell, $a=2.5 \mathrm{~nm}$; aspect ratio, $b=0.56$; alkyl shell width, $\delta=1.6 \mathrm{~nm}$; number of rosettes per toroid, $\left.N_{\mathrm{agg}}=100\right)$ suggest an internal circular void with radius $=8.4 \mathrm{~nm}$, which would certainly be capable of accommodating two fibers to generate nano-[n $>$ 2]catenanes. The comparison of the SAXS and SANS data, which highlight different parts of the structure $^{12}$, and associated analysis clearly point to the fibers having a core-shell structure, in line with being formed by stacking of the rosettes, with the alkyl region outside a more electron-dense core. These alkyl regions are highly penetrated by solvent molecules $(71 \%$ based on the SANS/SAXS analysis). Given the potential for alkyl chain overlap, it is therefore conceivable that the nano-[n $\geq$ 2]catenanes form by secondary nucleation of rosettes or monomers of $\mathbf{1}$ in these diffuse shells (Supplementary Discussion 2).

The isolated toroids are kinetically stable upon dilution to $c_{\mathrm{T}}=1 \times 10^{-6} \mathrm{M}$ at $353 \mathrm{~K}$, which is a prerequisite for their use as seeds. We thus added a small portion of the toroid solution to a large volume of monomer solution of $\mathbf{1}$ in $\mathrm{MCH}\left(c_{\mathrm{T}}=5 \times 10^{-6} \mathrm{M}\right)$ at $353 \mathrm{~K}$ and subsequently lowered the solution temperature to just below the elongation temperature $\left(T_{\mathrm{e}}=338 \mathrm{~K}\right.$, Fig. $\left.2 \mathrm{~g}\right)$. As shown in Fig. $2 \mathrm{~h}$ and Supplementary Fig. 9, the presence of toroids clearly accelerated the growth of an absorption band at $470 \mathrm{~nm}$, associated with the $\pi-\pi$ stacking of the diphenylnaphthalene cores ${ }^{16}$. As the elongation of the toroid seeds is topologically not possible due to lack of termini ${ }^{20-22}$, the above observation clearly demonstrates that the toroids promote the secondary nucleation of monomers of $\mathbf{1}$ on their surface. 
We further evaluated the reaction order associated with the secondary nucleation process of monomers by analyzing a set of aggregation kinetics data acquired for different concentrations of $\mathbf{1}$ in the absence of seeds (Fig. 2k). In protein aggregation, the time required to consume half of the total amount of monomers is referred to as the "half-time" $\left(t_{50}\right)$, and $t_{50}$ is related to the initial monomer concentration $\left(C_{0}\right)$ via the scaling law $t_{50} \propto\left(C_{0}\right)^{\gamma}$ where $\gamma$ is the scaling exponent ${ }^{19}$. In systems dominated by secondary nucleation processes, $\gamma$ is related to the reaction order $\left(n_{2}\right)$ according to:

$\gamma=-\left(1+n_{2}\right) / 2$

We thus plotted $\log \left(t_{50}\right)$ as a function of $\log C_{0}$ in order to evaluate $\gamma(\text { Fig. } 2 \mathrm{~m})^{19}$. From the slope, we found that $\gamma$ was equal to -1.7 , which corresponds to an $n_{2}$ value of 2.4 . This value is consistent with monomer-dependent secondary processes, such as those observed in the kinetics of amyloid growth ${ }^{19,25}$, suggesting that significant secondary nucleation occurs in the present system.

We used multiscale molecular simulations to study the event of a secondary nucleation occurring on the surface of pre-existing toroids (or oligomers) in $\mathrm{MCH}$. To deal with the complex hierarchical nature of the system, we developed coarse-grained (CG) models for the rosettes (Fig. 3a) reliably capturing their structural behavior and the rosette-rosette interactions in solution, while at the same time allowing us to study their assembly on a sufficiently large scale (see Supplementary Computational Details). The results of these simulations indicate that solvophobic interactions can make the self-assembly of rosettes more likely to initiate on the surface of preformed toroids. CG molecular dynamics simulations showed a strong propensity of the rosettes to axial stacking. However, it was also observed that these may interact with the surface of oligomers formed in the system, which is consistent with the experimentally suggested secondary nucleation on the toroids. To obtain a tangible evidence of such a scenario, we created a CG model of a prestacked rosettes having size consistent with the experimental one (blue part 
in Fig. 3a). A portion of this toroid was used in metadynamics simulations ${ }^{26}$ allowing to study the interaction of one single rosette or of a rosette trimer with the toroid surface ${ }^{27}$. In Fig. $3 \mathrm{~b}$, we plot the free-energy profile for the interaction between the rosette trimer and the toroid as a function of the distance between their centers (the same simulation with a single rosette provided analogous results). This shows strong affinity, suggesting that preassembled rosette toroids work as nucleation seeds for the growth of other ones onto their surface in $\mathrm{MCH}$. The formation of catenanes would then be related to the statistical probability for the toroids to close-up in such a way to form mechanically interlocked topologies (Fig. 1d).

Given that the solute-solute interactions that play a critical role in secondary nucleation are affected by the solvent ${ }^{28}$, we also evaluated the reaction order in $n$-octane as a representative $n$-alkane solvent (Fig. 21). The corresponding $\gamma(-2.9)$ and $n_{2}(4.8)$ values suggest a higher tendency toward secondary nucleation in $n$-octane (Fig. $2 \mathrm{~m}$ ). In line with this finding, much higher proportions of toroids were catenated when we used $n$-alkanes ( $n$-hexane to $n$-decane) as the poor solvent (Fig. $3 \mathrm{c}$ and Supplementary Figs. 3b, 10 and 11). It should be noted that the overall toroid yield decreased in linear alkane solvents compared to cyclic alkanes ( $\mathrm{MCH}$ and cyclohexane) because the open-elongation pathway becomes more feasible with decreasing solvent polarity (Fig. 3c and Supplementary Figs. 3a and 12). The use of longer $n$-alkanes ( $n$-undecane and $n$-dodecane) was not effective due to agglomeration of assemblies (Supplementary Fig. 13). Surprisingly, we found even longer congeners (Fig. 3d-f and Supplementary Fig. 14a) such as nano-[5]catenane in $n$-octane, which we coined "nanolympiadane" (length: $\sim 80 \mathrm{~nm}$; Supplementary Fig. 14b,c) in homage to the [5]catenane system first reported by Stoddart and colleagues ${ }^{10}$. Its topology, visualized by AFM, is impressively similar to the Olympic rings, although in our nanolympiadane, the five rings do not adopt the helical orientation seen in the Olympic logo (Fig. 3g and Supplementary Fig. 15), which is most likely due to the adsorption of the structure on the highly oriented pyrolytic graphite (HOPG) substrate surface. 
In order to prepare further elongated nanocatenanes, the mechanism for the spontaneous self-assembly of the aforementioned oligomeric nanocatenanes should be considered. Rowan et al. have recently reported the synthesis of poly[n]catenanes with $[4 \leq \mathrm{n} \leq 130]$ by interlocking 68 -membered macrocyclic ditopic ligands and a cyclizable threading molecule ${ }^{9}$. The metallo-supramolecular polymer templates, whose degree of polymerization practically determines the degree of polycatenation, were preassembled by the addition of $\mathrm{Zn}^{2+}$. In the present system, preassembly of such templates is obviously impossible, and elongation of the catenanes must occur in a stepwise manner (Fig. 4a). Accordingly, increasing local concentrations by injecting all the monomer at once would not be effective to increase [n]. Instead, a portion-wise injection protocol would more likely provide conditions in which monomers could be fed to preformed toroid seeds upon each injection in a living manner ${ }^{19,20}$. Furthermore, the portion-wise injection of monomer would probably result in lower local monomer concentration, which would facilitate secondary nucleation ${ }^{28-30}$ as well as reduce the occurrence of the unwanted open-elongation pathway leading to randomly coiled fibers.

We thus injected the monomer solution in ten portions (one $10 \mu \mathrm{L}$ injection per second; Fig. $4 \mathrm{~b}$ ). As shown in Fig. 4c and Supplementary Figs. 16 and 17, this approach increased both the overall toroid yield and the proportion of catenanes using $n$-alkane as well as cycloalkane solvents. For example, a ten-portion injection into $n$-octane provided a toroid yield of $49 \%$ based on the monomer (Supplementary Figs. 16 and 17), 41\% of which were catenated (Fig. 4c). The catenation number [n] was even more strongly affected than the overall catenane yield. Although "n" rarely exceeded 5 in single-injection experiments using $n$-alkanes, the ten-portion injection method produced nanopoly[n]catenanes whose "n" values beyond 7 (Fig. 4d-g and Supplementary Fig. 18). Fig. 4e-g show nano-poly[17], [18] and [22]catenanes. In these samples, the number of toroids that form nano-poly[n $\geq$ 3 ]catenanes is higher than that of nano-[2]catenanes (Fig. 4d). This result suggests that a cooperativity 
might be responsible for the polycatenation, presumably because two interlocked toroids can provide a specific nanospace that facilitates secondary nucleation more effectively than the surface of single

toroids. The elongated catenanes are stable in solution for months, suggesting that the toroidal constituents are kinetically stable against chain-breaking and interchain end-to-end connection as well as reorganization through monomer exchange.

We have demonstrated that the controlled use of secondary nucleation is an effective strategy to create mechanically interlocked nanostructures. Similar approaches could be applied to other macromolecular systems based on covalent or noncovalent polymerization. The purification of nanocatenanes is also an important challenge in order to investigate the physical properties of these topologically exotic molecular assemblies. We have already succeeded in separating toroid-based topologies (nano[n]catenanes and single toroids) from open-ended species via the thermal reorganization of the openended short fibers into elongated helical fibers and subsequent membrane filtration. We envisage that further catenation $(n>100)$ by enhancing the aforementioned cooperativity would allow us to purify those elongated species, which could lead to the discovery of unprecedented physical properties arising from mesoscopic topologies.

\section{Acknowledgements}

This work was supported by KAKENHI grant no. 26102010 and a Grant-in-Aid for Scientific Research on Innovative Areas “ $\pi$-Figuration” (grant no. 26102001) from the Japanese Ministry of Education, Culture, Sports, Science, and Technology (MEXT). This work was also supported by JSPS KAKENHI grant no. 19H02760. S.Y. acknowledges financial support from the Murata Science Foundation and the Shorai Foundation for Science and Technology. S.D. and K.A. thank the JSPS for a research fellowship. P19341 and 17J02520 are the corresponding fellowship IDs of S.D. and K.A., respectively. G.M.P. acknowledges the funding received by the Swiss National Science Foundation (SNSF grants 
IZLIZ2_183336 and 200021_175735) and by the European Research Council (ERC) under the European Union's Horizon 2020 research and innovation program (grant agreement no. 818776 DYNAPOL). G.M.P. also acknowledges the computational resources provided by the Swiss National Supercomputing Center (CSCS) and by CINECA.

\section{Author Contributions}

S.Y. and S.D. designed the project. Y.Ka. and S.H. performed most of the experimental works. M.J.H., A.J.S., R.D. and N.M. recorded and simulated the SAXS/SANS data and wrote the SAXS/SANS section of the manuscript. G.M.P., L.P. and C.P. performed the theoretical calculation and wrote the theoretical calculation section of the manuscript. S.Y. and S.D. analyzed and explained the experimental data. S.Y. and S.D. prepared the overall manuscript including figures. All authors including K.A., A.I., T.S., D.D.P., and Y.Ki. have contributed by commenting on the manuscript. The overall project was directed by S.Y.

\section{Data availability}

The authors declare that all other data supporting the findings of this study are available within the paper and its supplementary information files.

\section{Code availability}

There is no experiment requiring custom code or mathematical algorithm.

1. Segawa, Y. et al. Topological molecular nanocarbons: all-benzene catenane and trefoil knot. Science 365, 272-276 (2019).

2. Leigh, D. A., Pritchard, R. G. \& Stephens, A. J. A star of David catenane. Nat. Chem. 6, 978-982 (2014). 
3. Li, H. et al. Quantitative self-assembly of a purely organic three-dimensional catenane in water. Nat. Chem. 7, 1003-1008 (2015).

4. Fujita, M., Ibukuro, F., Hagihara, H. \& Ogura, K. Quantitative self-assembly of a [2]catenane from two preformed molecular rings. Nature 367, 720-723 (1994).

5. Hunter, C. A. Synthesis and structure elucidation of a new [2]-catenane. J. Am. Chem. Soc. 114, 5303-5311 (1992).

6. Ashton, P. R. et al. A [2]catenane made to order. Angew. Chem. Int. Ed. Engl. 28, 1396-1399 (1989).

7. Dietrich-Buchecker, C. O., Sauvage, J. P. \& Kintzinger, J. P. Une nouvelle famille de molecules: les metallo-catenanes. Tetrahedron Lett. 24, 5095-5098 (1983).

8. Wasserman, E. Chemical topology. Sci. Am. 207, 94-102 (1962).

9. Wu, Q. et al. Poly[n]catenanes: synthesis of molecular interlocked chains. Science 358, 1434-1439 (2017).

10. Amabilino, D. B., Ashton, P. R., Reder, A. S., Spencer, N. \& Stoddart, J. F. Olympiadane. Angew. Chem. Int. Ed. Engl. 33, 1286-1290 (1994).

11. Yagai, S., Kitamoto, Y., Datta, S. \& Adhikari, B. Supramolecular polymers capable of controlling their topology. Acc. Chem. Res. 52, 1325-1335 (2019).

12. Hollamby, M. J. et al. Simultaneous SAXS and SANS analysis for the detection of toroidal supramolecular polymers composed of noncovalent supermacrocycles in solution. Angew. Chem. Int. Ed. 55, 9890-9893 (2016).

13. Yagai, S. et al. Self-organization of hydrogen-bonding naphthalene chromophores into J-type nanorings and H-type nanorods: impact of regioisomerism. Angew. Chem. Int. Ed. 51, 6643-6647 (2012). 
14. Adhikari, B., Aratsu, K., Davis, J. \& Yagai, S. Photoresponsive circular supramolecular polymers: a topological trap and photoinduced ring-opening elongation. Angew. Chem. Int. Ed. 58, 3764-3768 (2019).

15. Adhikari, B. et al. Light-induced unfolding and refolding of supramolecular polymer nanofibres. Nat. Commun. 8, 15254 (2017).

16. Prabhu, D. D. et al. Self-folding of supramolecular polymers into bioinspired topology. Sci. Adv. 4, eaat8466 (2018).

17. Korevaar, P. A. et al. Pathway complexity in supramolecular polymerization. Nature 481, $492-496$ (2012).

18. Gil-Ramírez, G., Leigh, D. A. \& Stephens, A. J. Catenanes: fifty years of molecular links. Angew. Chem. Int. Ed. 54, 6110-6150 (2015).

19. Cohen, S. I. A., Vendruscolo, M., Dobson, C. M. \& Knowles, T. P. J. From macroscopic measurements to microscopic mechanisms of protein aggregation. J. Mol. Biol. 421, 160-171 (2012).

20. Ogi, S., Sugiyasu, K., Manna, S., Samitsu, S. \& Takeuchi, M. Living supramolecular polymerization realized through a biomimetic approach. Nat. Chem. 6, 188-195 (2014).

21. Wang, X. et al. Cylindrical block copolymer micelles and co-micelles of controlled length and architecture. Science 317, 644-647 (2007).

22. Zhang, W. et al. Supramolecular linear heterojunction composed of graphite-like semiconducting nanotubular segments. Science 334, 340-343 (2011).

23. Suzuki, A. et al. Topological impact on the kinetic stability of supramolecular polymers. J. Am. Chem. Soc. 141, 13196-13202 (2019).

24. Breßler, I., Kohlbrecher, J. \& Thünemann, A. F. SASfit: a tool for small-angle scattering data analysis using a library of analytical expressions. J. Appl. Cryst. 48, 1587-1598 (2015). 
25. Meisl, G. et al. Molecular mechanisms of protein aggregation from global fitting of kinetic models. Nat. Protoc. 11, 252-272 (2016).

26. Laio, A. \& Parrinello, M. Escaping free-energy minima. Proc. Natl Acad. Sci. USA 99, 1256212566 (2002).

27. Bochicchio, D., Salvalaglio, M. \& Pavan, G. M. Into the dynamics of a supramolecular polymer at submolecular resolution. Nat. Commun. 8, 147 (2017).

28. Anwar, J., Khan, S. \& Lindfors, L. Secondary crystal nucleation: nuclei breeding factory uncovered. Angew. Chem. Int. Ed. 54, 14681-14684 (2015).

29. Kondepudi, D. K. \& Asakura, K. Chiral autocatalysis, spontaneous symmetry breaking, and stochastic behavior. Acc. Chem. Res. 34, 946-954 (2001).

30. Kondepudi, D. K., Kaufman, R. J. \& Singh, N. Chiral symmetry breaking in sodium chlorate crystallizaton. Science 250, 975-976 (1990). 

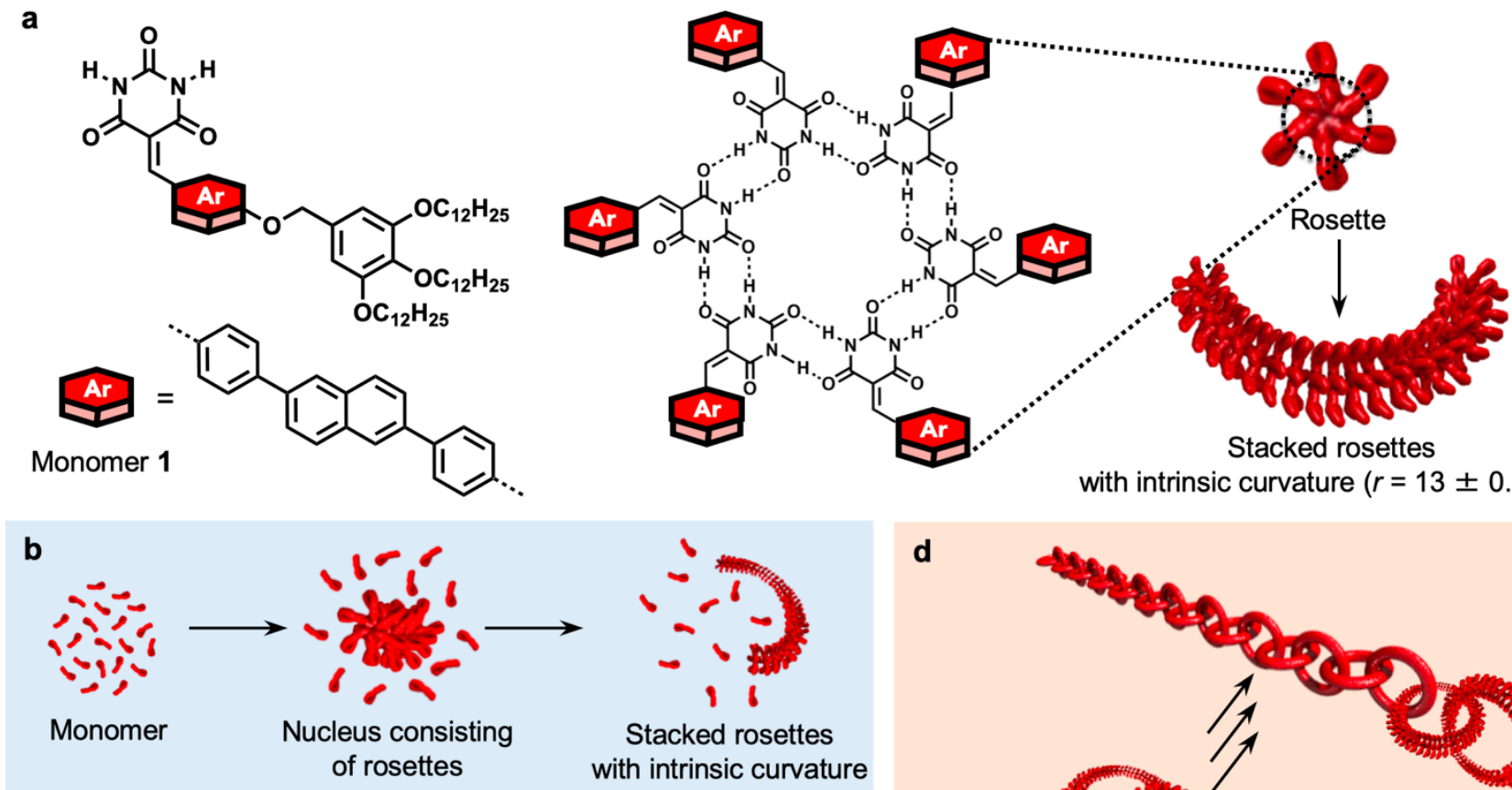

d
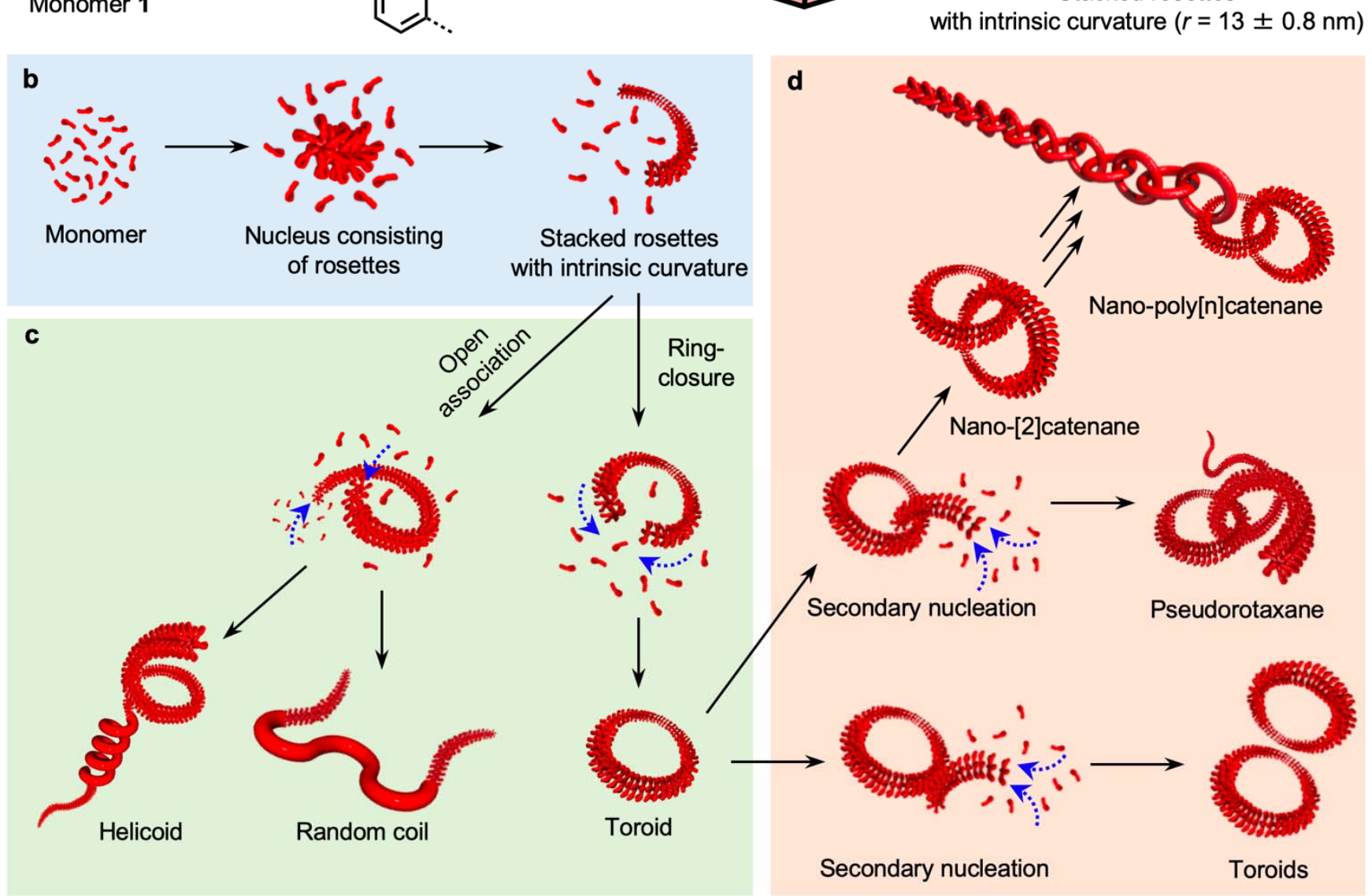

Figure 1 | Self-assembly of a barbiturated monomer into various supramolecular polymer

topologies. a, Chemical structure of barbiturated naphthalene derivative $\mathbf{1}$ and a schematic

representation of the unique stacking of its hydrogen-bonded cyclic hexamers (rosettes) to generate an intrinsic curvature. b, c, Schematic illustration of the primary nucleation (b) and elongation (c) of the monomers into distinct nanotopologies. $\mathbf{d}$, Schematic illustration of secondary nucleation on a toroid to produce interlocked topologies. 

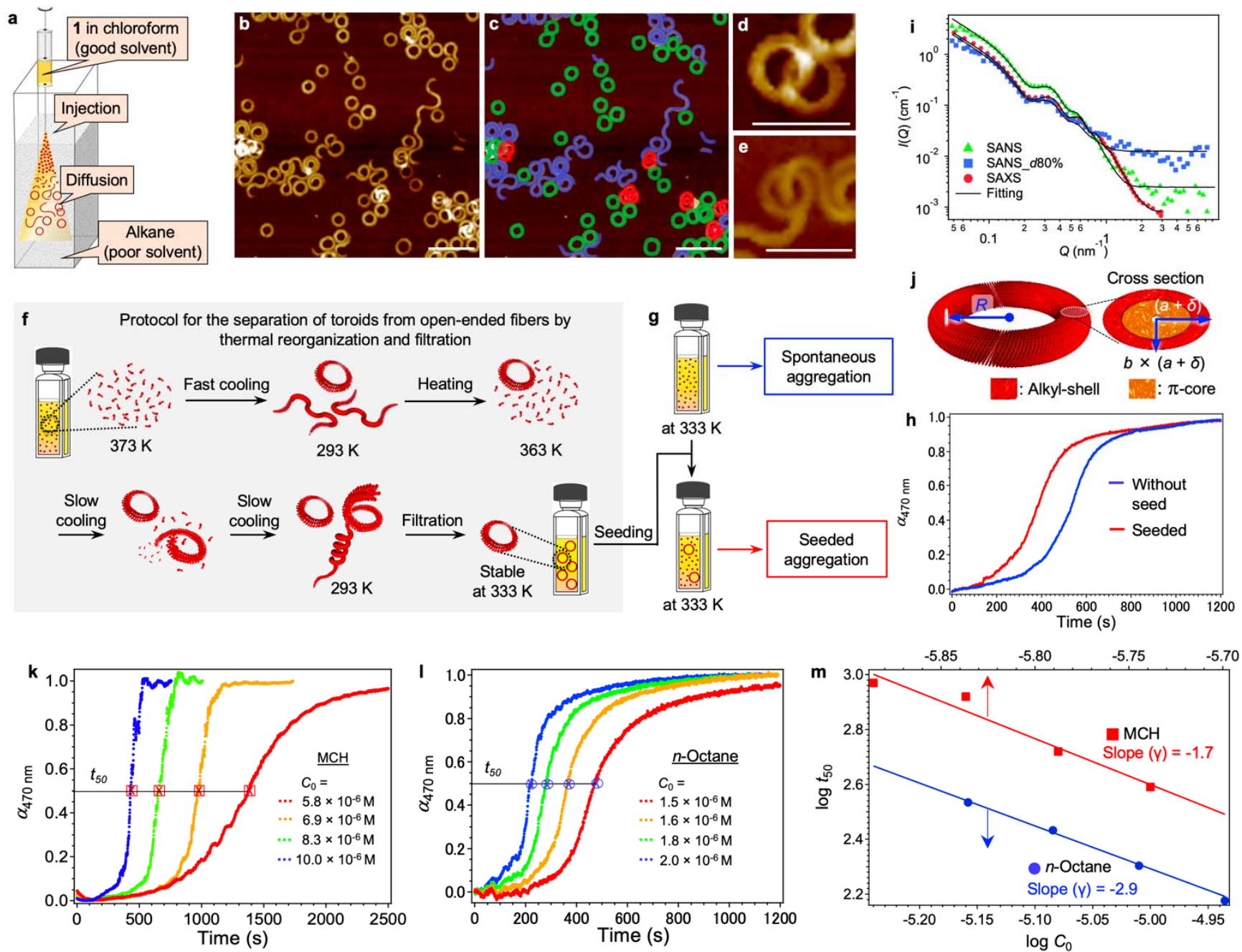

Figure 2 | Supramolecular polymerization protocols. a, Depiction of the solvent-mixing protocol for

the preparation of kinetic supramolecular polymer species. b-e, AFM image of supramolecular polymers obtained by injecting a $100 \mu \mathrm{L}$ chloroform solution of $\mathbf{1}\left(c_{\mathrm{T}}=1 \times 10^{-3} \mathrm{M}\right)$ into $900 \mu \mathrm{L}$ of $\mathrm{MCH}$ via a single injection; scale bars $=100(\mathbf{b}$ and $\mathbf{c})$ and $50(\mathbf{d}$ and $\mathbf{e}) \mathrm{nm}$. c, a complex mixture; d, nano-[2]catenane; $\mathbf{e}$, pseudorotaxane. Image $\mathbf{c}$ is a duplicate of image $\mathbf{b}$, but different topologies are colored to estimate the relative area (see Supplementary Information): blue for open-ended coils; green for single toroids; red for mechanically interlocked toroids. f, Protocol for separating toroids from openended fibers via thermal reorganization followed by membrane filtration. g, Protocol for the seeded supramolecular polymerization using the isolated toroids as seeds. $\mathbf{h}$, Comparison of the time-dependent changes in the molar fractions of aggregates of $\mathbf{1}\left(\alpha_{470 \mathrm{~nm}}\right.$, monitored at $\left.\lambda=470 \mathrm{~nm}\right)$ in a monomeric $\mathrm{MCH}$ solution $\left(c_{\mathrm{T}}=4.8 \times 10^{-6} \mathrm{M}\right)$ at $333 \mathrm{~K}$ in the presence (red dots) and absence (blue dots) of toroid 
seeds $\left([\right.$ seed $]=1.6 \times 10^{-6} \mathrm{M}$ based on the monomers after solvent mixing). $\mathbf{i}$, SANS (green triangles and blue squares) and SAXS (red circles) data of $\mathbf{1}\left(c_{\mathrm{T}}=5 \times 10^{-4} \mathrm{M}\right)$ in $\mathrm{MCH}-d_{14}$ (green triangles) and a mixed solvent comprising $80 \% \mathrm{MCH}-d_{14}$ and $20 \% \mathrm{MCH}-h_{14}$ (blue squares). Black solid lines represent fits of the data, using a toroid form factor and a flat background. $\mathbf{j}$, Representation of the core (orange)shell (red) toroid of $\mathbf{1}$ with parameters obtained from model fitting of the SAS data $\left(R, a, \delta_{\text {shell }}, b\right)$. k, $\mathbf{l}$, Time-dependent changes in the molar fractions of aggregates of $\mathbf{1}$ at various $C_{0}$ in $\mathrm{MCH}(\mathbf{k})$ and $n$ octane (l) at $333 \mathrm{~K}$ in the absence of toroid seeds. $\mathbf{m}$, Log-log plot of the time required for $C_{0}$ to drop by $50 \%$ in the seeded experiments in $\mathrm{MCH}$ (red squares) and $n$-octane (blue circles) as a function of $C_{0}$. 

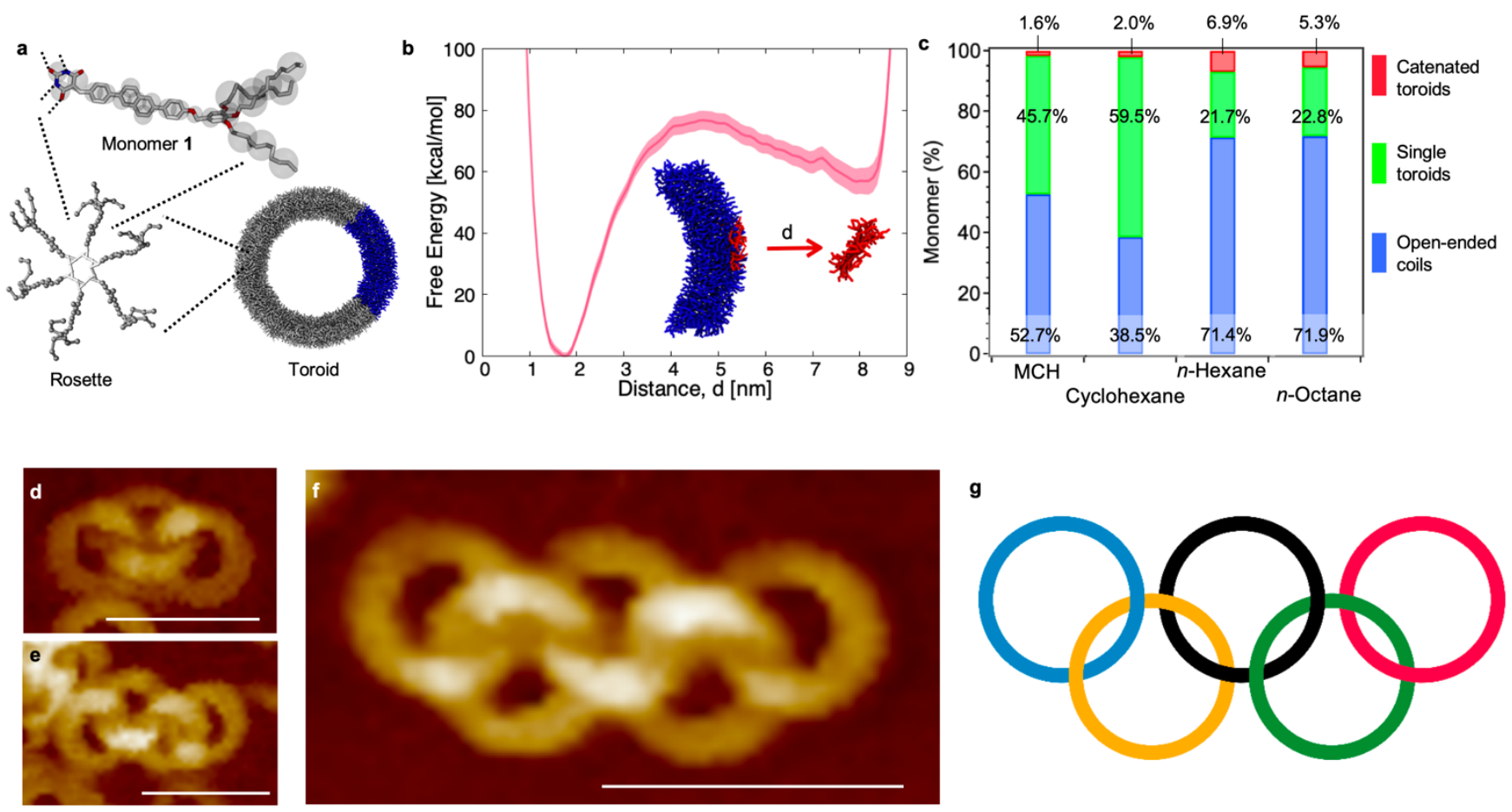

Figure 3 | Solvent dependence of the catenane yield. a, All atom (AA) and coarse-grained (CG) models of one arm, one rosette (hexagonal assembly of six arms) and one toroid of $\mathbf{1}$ used in the simulations. b, Free-energy profile for the interaction between one rosette trimer and the surface of the toroid (a portion of a full toroid composed of 50 rosettes) as a function of the distance between the center of the toroid section (blue) and the center of the rosette trimer (red). c, Histogram showing the yield of catenanes (red bar), toroids (green bar), and random coils (blue bar) depending on the choice of poor solvent in single injection solvent mixing experiment. d-f, AFM images of nano-[3]catenane (d), nano-[4]catenane (e), and nano-[5]catenane (f) obtained by injecting $100 \mu \mathrm{L}$ of a chloroform solution of $\mathbf{1}\left(1 \times 10^{-3} \mathrm{M}\right)$ into $900 \mu \mathrm{L}$ of $n$-octane in one portion; scale bars $=50 \mathrm{~nm}$. g, Symbol of the Olympic rings; reproduced with permission from the International Olympic Committee. 
a

Preorganized polycatenation

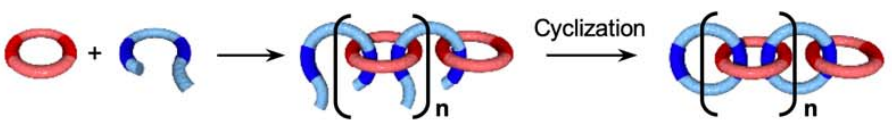

Precursors

Preorganized polymer

Polycatenanes
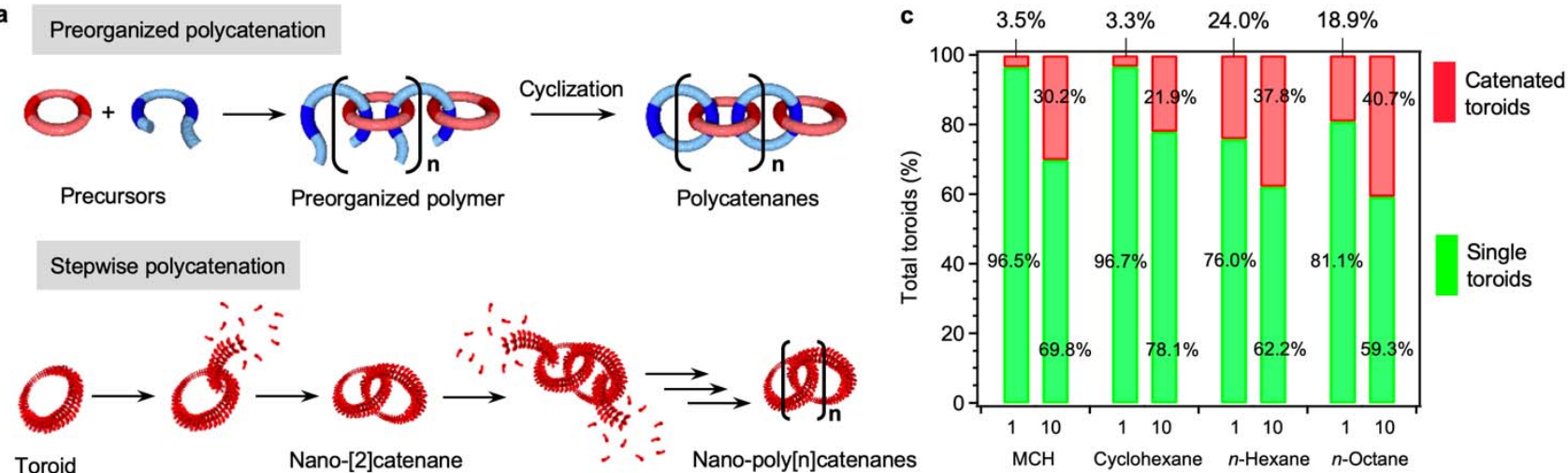
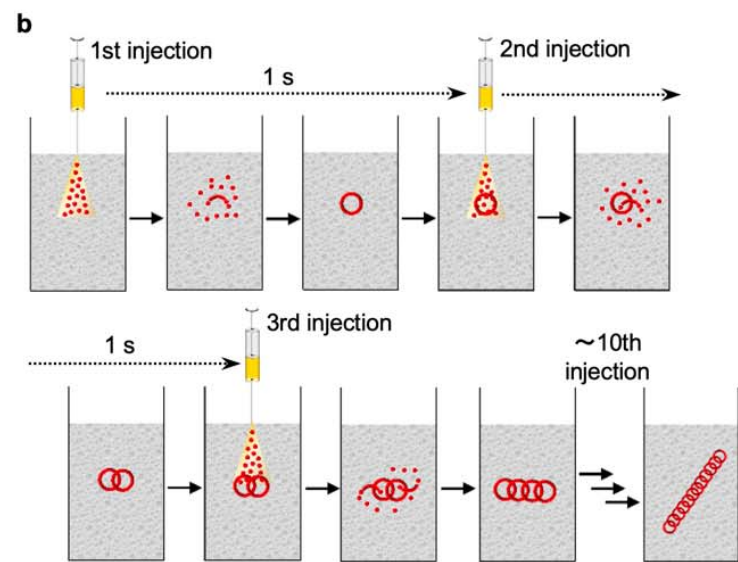

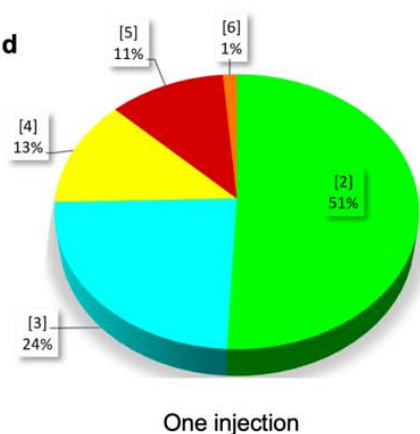

(Total number of interlocked toroids $=441$ )

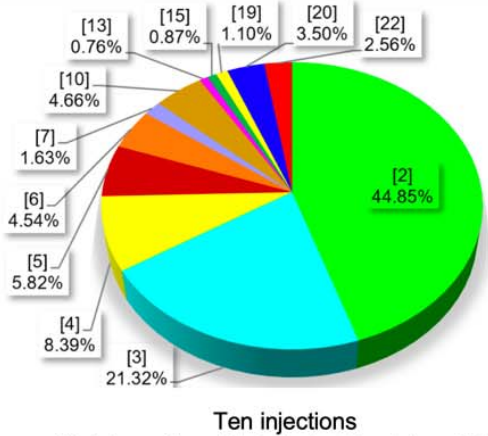

(Total number of interlocked toroids $=1717)$

$$
\begin{aligned}
& \text { Percentage }(\%) \text { of toroids } \\
& \text { involved in nano-[n]catenane }
\end{aligned}=\frac{n \times \text { Number of nano-[n]catenane }}{\text { Total number of interlocked toroids }} \times 100
$$
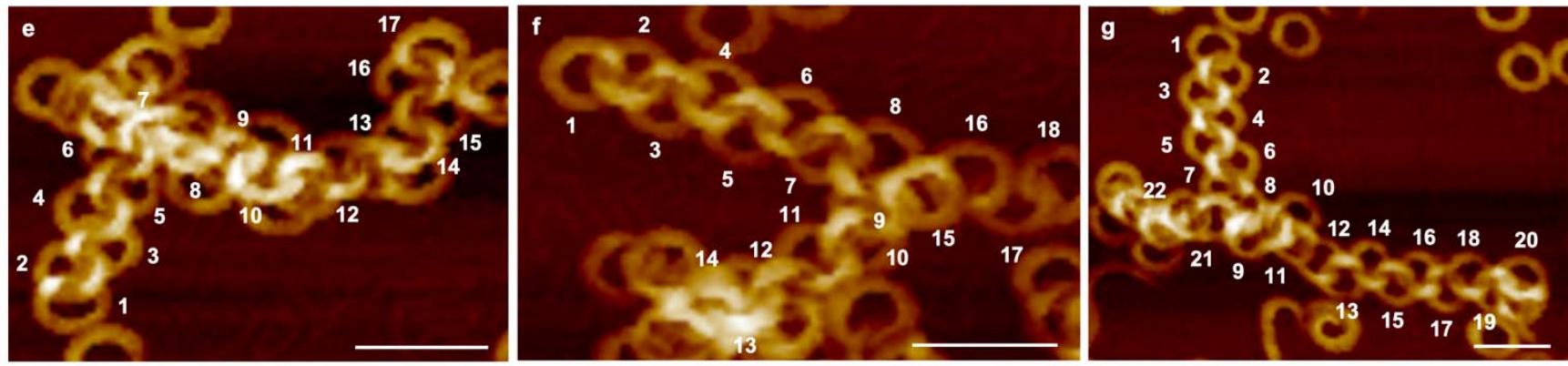

Figure 4 | Nano-polycatenanes. a, Schematic illustration of polycatenation by preorganization and stepwise manner. $\mathbf{b}$, Depiction of a ten-injection solvent-mixing experiment. c, Histogram comparing the yields of interlocked toroids (red bar) and single toroids (green bar) with respect to the overall toroids for different poor solvents and number of monomer solution injections. d, Pie charts showing the percent interlocked toroids constituting various nano-[n]catenanes for one hundred $500 \times 500 \mathrm{~nm}^{2}$ AFM images acquired after the one-injection (left) and ten-injections (right) solvent-mixing experiments with chloroform and $n$-octane as the good and poor solvent, respectively $\left(c_{\mathrm{T}}=1 \times 10^{-4} \mathrm{M}\right.$ after solvent mixing). e-g, AFM images of nano-poly[17]catenane (e), nano-poly[18]catenane (f), and nano- 
poly[22]catenane (g) obtained by injecting ten portions (one per second) of $10 \mu \mathrm{L}$ of a chloroform solution of $\mathbf{1}\left(1 \times 10^{-3} \mathrm{M}\right)$ into $900 \mu \mathrm{L}$ of poor solvent. nano-poly[17]catenane (e) was obtained when $n$ hexane was used as the poor solvent; nano-poly[18] catenanes (f) and nano-poly[22]catenanes (g) were obtained using $n$-octane as the poor solvent; inset numbering shows counting of interlocked toroids of nano-poly[n]catenanes; scale bars $=50 \mathrm{~nm}$. For these catenanes, branched catenation likely occurred at for example, toroid \#7 and \#13 in $\mathbf{e , ~} \# 9$ in $\mathbf{f}$, and \#8 in $\mathbf{g}$ via threading of a supramolecular polymer fiber through an already-catenated toroid, which would seem to be sterically challenging unless the fibers grow in an organized fashion from secondary nuclei generated at the inner surface of the preformed catenanes. 